\title{
L1-induced Performance Analysis for Positive Systems with Interval Uncertainties \\ Xiaoming Chen
}

\author{
College of Automation Engineering, Nanjing University of Aeronautics and Astronautics, Nanjing, \\ 210016, China \\ xmchen@nuaa.edu.cn
} Keywords: Interval uncertainties; Iterative algorithm; linear Lyapunov functions; L1-induced
performance; Positive systems

\begin{abstract}
The $L_{1}$ performance analysis is studied for continuous-time interval positive systems. In this paper, necessary and sufficient conditions for stability and $L_{1}$ performance are established for positive linear systems through linear inequalities.
\end{abstract}

\section{Introduction}

In real world, positive systems exist in many branches of technology and science such as industrial engineering [1], ecology [2]. For non-negative initial conditions and inputs, the variables of positive systems always take non-negative values. Positive systems are special systems and they are defined on cones instead of linear spaces. Therefore, many well-established approaches to system analysis and synthesis cannot give satisfactory results, which motivates the research on positive systems.

Recently, many research results on positive systems have been reported in the literature [4-11] after the development of a system-theoretic approach to positive systems in [3]. For example, controllability and reachability for positive systems have been studied in [14] and [15]. The positive state-space representation for a given transfer function has been proposed in [12]. For non-negative and time-delay compartmental dynamic systems, stability has been thoroughly studied in [18-19]. Research on 2-D positive systems has appeared in [7]. Conditions for positive realizability in terms of convex analysis have been presented in [13]. The design of state-feedback controllers guaranteeing the closed-loop system to be asymptotically stable has been studied by the linear programming approach in [16]. Moreover, the problem of controller design for positive systems has been investigated by the linear matrix inequality (LMI) approach in [17].

In recent years, many novel results through linear Lyapunov functions have appeared [17]. As is well known, a lot of existing results about positive systems are derived via quadratic Lyapunov functions and many effective methods are available. In comparison with the quadratic Lyapunov function based approach, the novel method is more simple and amenable to computation and analysis. The reason we use the novel approach is that the variables of positive systems are non-negative and therefore a linear Lyapunov function becomes valid.

As is well known, it is inevitable that uncertainties enter the system parameters due to the fact that practical systems are usually influenced by variations, disturbances or environmental perturbations. For interval positive systems, the L1-induced performance analysis deserves to be studied and is challenging, due to the complexity caused by parameter uncertainties.

In this paper, the L1-induced performance analysis problem is considered for interval positive systems. In detail, for a given positive system, the objective is to propose an L1-induced performance index. In Section 2, the L1-induced performance is first established and some preliminary results are proposed. In Section 3, a performance characterization is derived, under which the positive system is robustly stable with the L1-induced performance. We present the conclusion of this paper in Section 4. 


\section{Preliminaries}

In this section, many results are introduced for positive systems. First, we present the following notations.

R denotes the set of real numbers; $A \geq \geq B$ (respectively, $A>>B$ ) means that $A-B \geq \geq 0$ (respectively, $A-B>>0$ ). For $A \in \mathrm{R}^{m \times n}$, aij is the element located at the $i$ th row and the $j$ th column. The notation $A \geq \geq B$ (respectively, $A>>B$ ) means that the matrix $A-B \geq \geq 0$ (respectively, $A-B>>0$ ). The $L 1$-norm of $x$ is defined as $\|x\|_{L 1}=\int\|x(t)\| 1 d t$. We denote $\mathbf{1}=$ $[1,1, \ldots, 1]^{T}$. The induced 1-norm of a matrix $Q=[q i j] \in \mathrm{R}^{m \times n}$ is denoted by $\|Q\| 1=\max \left(\sum\right.$ $|q i j|) . A \in \mathrm{R}^{n \times n}$ is Metzler, if all its off-diagonal elements are positive. That is, $\forall(i$, $j$ ) , $i \neq j, a i j \geq 0$. $A \geq \geq 0$ (respectively, $A>>0$ ) means that for all $i$ and $j, a_{i j} \geq 0$ (respectively, $\left.a_{i j}>0\right) . \mathrm{R}^{n}$ denotes the set of $n$-column real vectors. The induced 1-norm of a matrix $Q=[q i j]$ $\in \mathrm{R}^{m \times n}$ is denoted by $\|Q\| 1=\max \left(\sum\left|q_{i j}\right|\right)$. For matrices $A, \underline{A}, A \in \mathrm{R}^{n \times m}$, the notation $A \in$ $[\underline{A}, A]$ means that $\underline{A} \leq \leq A \leq \leq A$. The symbol $\rho(A)$ denotes the spectral radius of matrix $A$, that is, $\rho(A)=\max \{|\lambda i(A)|, i=1,2, \ldots, n\}$. The symbol coli(A) denotes the $i$ th column of matrix $A$. The $L 1$-norm of $x$ is defined as $\|x\| L 1=\int\|x(t)\| 1 d t$. We denote $1=[1,1, \ldots, 1]^{T} . A \geq \geq 0$ (respectively, $A>>0$ ) means that for all $i$ and $j, a_{i j} \geq 0$ (respectively, $a_{i j}>0$ ). The superscript “ $T$ ” denotes matrix transpose. $\mathrm{R}^{n \times m}$ is the set of all real matrices of dimension $n \times m$. $\|\cdot\|$ represents the Euclidean norm for vectors. The 1-norm of a vector $x(t)=(x 1(t), x 2(t), \ldots$, $\left.x_{n}(t)\right)$ is defined as $\|x(t)\| 1=\sum|x i(t)| . \mathrm{N}$ is the set of natural numbers. The spectral abscissa of matrix $A$ is denoted by $\alpha(A)=\max \{\operatorname{Re} \lambda i(A), i=1,2, \ldots, n\}$.

Consider a system:

$$
\begin{aligned}
& \mathrm{x}(\mathrm{t})=\mathrm{Ax}(\mathrm{t})+\mathrm{Bu}(\mathrm{t})+\mathrm{B}_{\mathrm{w}} \mathrm{w}(\mathrm{t}), \\
& \mathrm{z}(\mathrm{t})=\mathrm{C}_{\mathrm{z}} \mathrm{x}(\mathrm{t})+\mathrm{Du}(\mathrm{t})+\mathrm{D}_{\mathrm{zw}} \mathrm{w}(\mathrm{t}), \\
& \mathrm{y}(\mathrm{t})=\mathrm{Cx}(\mathrm{t}),
\end{aligned}
$$

where $x(t) \in R^{n}, \mathrm{u}(t) \in R^{n} w(t) \in R^{m}, y(t) \in R^{r}, z(t) \in R^{q}$ are the state, control input, disturbance input, measurement and controlled output, respectively.

The following definition is introduced which will be used in the paper.

Definition 1 The continuous-time linear system (1) is said to be positive if for all initial state $x(0)$ $\geq \geq 0$ and disturbance input $w(t) \geq \geq 0$, we have $x(t) \geq \geq 0, z(t) \geq \geq 0$ and $y(t) \geq \geq 0$ for $t>0$.

Next, some useful results are introduced which will be used later in this paper.

Proposition 1 For a positive system (1), we say it is stable if and only if there exists a nonnegative vector $p$ satisfying the following inequality:

$$
p^{T} A \leq \leq 0 .
$$

Lemma 1 The continuous-time system (1) is positive if and only if $A$ is M-matrix, $B w, C z, D z w$, and $C$ are all nonnegative.

Then, the definition of $L_{1}$-induced norm is proposed in the following.

For a stable positive system in (1) with zero initial conditions, it has $L_{1}$-induced performance at the level $\gamma$ if,

$$
\|Z\|_{L_{1}}<\gamma\|w\|_{L_{1}}
$$

under zero initial conditions.

\section{Main Results}

The following theorem is proposed and it serves as a characterization on the stability and performance of system (1). 
Theorem 1. For a positive system in (1), it is stable with the performance in (2) satisfied under initial conditions if and only if we can find a nonnegative vector $p$ satisfying

$$
\left[\begin{array}{cc}
p^{T} & 1^{T}
\end{array}\right]\left[\begin{array}{cc}
A & B_{w} \\
C_{z} & D_{z w}-\gamma I
\end{array}\right]<<0 .
$$

Next, the following system is considered:

$$
\begin{aligned}
& \dot{x}(t)=A_{I} x(t)+B_{I u}(t)+B_{I} w(t), ? \\
& z(t)=C_{I z} x(t)+D_{I z} u(t)+D_{I z w} w(t), \bar{?} \\
& y(t)=C_{I} x(t), ?
\end{aligned}
$$

with

$$
A_{I} \in\left[\underline{A_{I}}, \overline{A_{I}}\right], B_{I w} \in\left[\underline{B_{I w}}, \overline{B_{I W}}\right], C_{I z} \in\left[\underline{C_{I z}}, \overline{C_{I z}}\right], D_{I z w} \in\left[\underline{D_{I z w}}, \overline{D_{I z w}}\right], C_{I} \in\left[\underline{C}_{I}, \overline{C_{I}}\right]
$$

Next, one definition about interval positive system is presented.

Definition 2. The interval positive system in (4) is robustly stable if it is stable over the whole uncertainty domain in (6).

Then, the following theorem is established as the performance characterization result for interval positive system in (4).

Theorem 2. The interval positive system in (4) is robustly stable and satisfies the $L_{1}$-induced performance for any $A_{I} \in\left[\underline{A_{I}}, \overline{A_{I}}\right], B_{I w} \in\left[\underline{B_{I w}}, \overline{B_{I w}}\right], C_{I z} \in\left[\underline{C_{I z}}, \overline{C_{I z}}\right], D_{I z w} \in\left[\underline{D_{I z w}}, \overline{D_{I z w}}\right], C_{I} \in\left[\underline{C}_{I}, \overline{C_{I}}\right]$ if and only if there exists a nonnegative vector $p$ satisfying the following inequalities:

$$
\left[\begin{array}{ll}
p^{T} & 1^{T}
\end{array}\right]\left[\begin{array}{lc}
\bar{A}_{I} & \bar{B}_{I w} \\
\bar{C}_{I z} & \bar{D}_{I z w}-\gamma I
\end{array}\right]<<0
$$

Proof:

For any $A_{I} \in\left[\underline{A}_{I}, \overline{A_{I}}\right], B_{I w} \in\left[\underline{B_{I w}}, \overline{B_{I w}}\right], C_{I z} \in\left[\underline{C_{I z}}, \overline{C_{I z}}\right], D_{I z w} \in\left[\underline{D_{I z w}}, \overline{D_{I z w}}\right], C_{I} \in\left[\underline{C_{I}}, \overline{C_{I}}\right]$, we have

$$
\begin{aligned}
& {\left[\begin{array}{ll}
p^{T} & 1^{T}
\end{array}\right]\left[\begin{array}{c}
A \\
C_{z}
\end{array}\right] \leq \leq\left[\begin{array}{ll}
p^{T} & 1^{T}
\end{array}\right]\left[\begin{array}{c}
\bar{A}_{I} \\
\bar{C}_{I z}
\end{array}\right]<0,} \\
& {\left[\begin{array}{ll}
p^{T} & 1^{T}
\end{array}\right]\left[\begin{array}{c}
B_{w} \\
D_{z w}-\gamma I
\end{array}\right] \leq \leq\left[\begin{array}{ll}
p^{T} & 1^{T}
\end{array}\right]\left[\begin{array}{c}
\bar{B}_{I w} \\
\bar{D}_{I z w}-\gamma I
\end{array}\right],}
\end{aligned}
$$

Therefore, it is noted that the interval system in (4) is robustly stable and satisfies the $L_{1}$-induced performance under initial conditions. The sufficiency is proved.

Next, we assume that the interval system in (4) is robustly stable and satisfies the $L_{1}$-induced performance under initial conditions. The following inequalities hold.

Then, the inequality (6) naturally holds.

$$
\left[\begin{array}{cc}
p^{T} & 1^{T}
\end{array}\right]\left[\begin{array}{cc}
A & B_{w} \\
C_{z} & D_{z w}-\gamma I
\end{array}\right]<<0
$$




\section{Summary}

In this paper, the $L_{1}$-induced performance analysis problem has been studied for interval positive systems. We have established a novel performance characterization to guarantee that the interval positive system is robust stable and satisfies the $L_{1}$-induced performance.

\section{References}

[1]. H. Caswell, Matrix Population Models: Construction, Analysis and Interpretation. (Sunderland, MA: Sinauer Assoc.,(2001)

[2]. J. Shen, J. Lam, Decay rate constrained stability analysis for positive systems with discrete and distributed delays. Syst. Sci. \& Control Eng. 2 (1), 7-12 (2014)

[3]. M.P. Fanti, B. Maione, B. Turchiano, Controllability of multi-input positive discrete-time systems. Int. J. Control 51 (6), 1295-1308 (1990)

[4]. Y. Ebihara, D. Peaucelle, D. Arzelier, LMI approach to linear positive system analysis and synthesis. Syst. \& Control Lett. 63 (1), 50-56 (2014)

[5]. J. Shen and J. Lam. On static output-feedback stabilization for multi-input multi-output positive systems. International Journal of Robust and Nonlinear Control, 25(16):3154 - 3162, (2015).

[6]. P. Li, J. Lam, Z. Shu, H1 positive filtering for positive linear discrete-time systems: An augmentation approach. IEEE Trans. Automat. Control 55 (10), 2337-2342 (2010)

[7]. L. Farina, On the existence of a positive realization. Syst. \& Control Lett. 28 (4), 219-226 (1996)

[8]. T. Kaczorek, Positive 1D and 2D Systems. (Germany, Berlin, Springer-Verlag, 2002)

[9]. J. Shen, J. Lam, H1 model reduction for positive fractional order systems. Asian J. Control 16 (2), 441-450 (2014)

[10]. W. Haddad, V. Chellaboina, Stability theory for nonnegative and compartmental dynamical systems with time delay. Syst. \& Control Lett. 51 (5), 355-361 (2004)

[11]. L. Benvenuti, L. Farina, A tutorial on the positive realization problem. IEEE Trans. Automat. Control 49 (5), 651-664 (2004)

[12]. E. Fornasini, M. Valcher, Linear copositive Lyapunov functions for continuous-time positive switched systems. IEEE Trans. Automat. Control 55 (8), 1933-1937 (2010)

[13]. D.G. Luenberger, Introduction to Dynamic Systems: Theory, Models, and Applications. (Wiley, New York, 1979)

[14]. X. Chen, J. Lam, and H. K. Lam. Positive filtering for positive Takagi-Sugeno fuzzy systems under $\ell 1$ performance. Information Sciences, 299:32 - 41, (2015).

[15]. J. Lian and J. Liu. New results on stability of switched positive systems: an average dwell-time approach. IET Control Theory \& Applications, 7(12):1651 - 1658,(2013).

[16]. X. Liu and J. Lam. Relationships between asymptotic stability and exponential stability of positive delay systems. International Journal of General Systems, 42(2):224 - 238, (2013).

[17]. J. Zhang and Y. Wang. Stability analysis of switched positive linear systems with stable and unstable subsystems. International Journal of Systems Science, 45(12):2458 - 2465, (2014).

[18]. X. Chen, J. Lam, and P. Li. Positive filtering for continuous-time positive systems under L1 performance. Int. J. Control, 87(9):1 - 8, (2014).

[19]. M.E. Valcher, Controllability and reachability criteria for discrete time positive systems. Int. J. Control 65 (3), 511-536 (1996). 